\title{
Well-posedness of a ratio-dependent Lotka-Volterra system with feedback control
}

\author{
Changyou Wang ${ }^{1,2,3^{*}}$, Yuqian Zhou', Yonghong Li ${ }^{2}$ and Rui Li ${ }^{3}$
}

\begin{tabular}{l}
\hline${ }^{\text {"Correspondence: }}$ \\
wangchangyou417@163.com \\
${ }^{1}$ College of Applied Mathematics, \\
Chengdu University of Information \\
Technology, Chengdu, P.R. China \\
${ }^{2}$ College of Science, Chongqing \\
University of Posts and \\
Telecommunications, Chongqing, \\
P.R. China \\
Full list of author information is \\
available at the end of the article
\end{tabular}

available at the end of the article

\begin{abstract}
The main purpose of this article is to consider a Lotka-Volterra predator-prey system with ratio-dependent functional responses and feedback controls. By using a comparison theorem and constructing a suitable Lyapunov function as well as developing some new analysis techniques, we establish a set of easily verifiable sufficient conditions which guarantee the permanence of the system and the global attractivity of a positive solution for the predator-prey system. Furthermore, some conditions for the existence, uniqueness, and stability of a positive periodic solution for the corresponding periodic system are obtained by using the fixed point theory and some new analysis method. In additional, some numerical solutions of the equations describing the system are given to verify that the obtained criteria are new, general, and easily verifiable.
\end{abstract}

Keywords: Predator-prey system; Feedback control; Permanence; Attractivity; Periodic solution

\section{Introduction}

After the pioneering works of Lotka and Volterra, a lot of work has been carried out on the predator-prey model. The most crucial element in these models is the "functional response"-the expression that describes the rate at which the number of prey is consumed by a predator. Modifications were limited to replacing the Malthusian growth function, the predator per capita consumption of prey functions such as Holling type I, II, III functional responses, or density-dependent mortality rates. These functional responses depend only on the prey volume, but soon it became clear that the predator volume can influence this function by direct interference while searching or by pseudo interference [1-3]. A simple way of incorporating predator dependence in the functional response was proposed by Arditi and Ginzburg [4], who first considered this response function as a function of the ratio. Moreover, Jost et al. [5] showed that prey-dependent and ratiodependent models can fit well with the time series generated by each other. Interestingly, it has been investigated that the ratio-dependent predator-prey models are more appropriate for predator-prey interactions when the predator involves serious hunting processes, like animals searching for animals, etc. [6-8]. It is justified through some basic but different principles that ratio-dependent models are more appropriate for modeling predator-prey interactions [9]. Kesh et al. [10] consider a food web model consisting of two competing prey and one predator population with predator interference. From the

(c) The Author(s) 2018. This article is distributed under the terms of the Creative Commons Attribution 4.0 International License (http://creativecommons.org/licenses/by/4.0/), which permits unrestricted use, distribution, and reproduction in any medium, provided you give appropriate credit to the original author(s) and the source, provide a link to the Creative Commons license, and indicate if changes were made. 
knowledge of saturated equilibria and by constructing the average Lyapunov function, the sufficient condition for permanent coexistence and extinction of species of the system is obtained. Pang and Wang [11] study a class of two-predator-one-prey ecosystem and show that the unique positive equilibrium solution of the system is globally asymptotically stable. Baek et al. [12] study a $x_{1}(t)$ elliptic system with ratio-dependent functional responses. By employing a comparison argument for the elliptic problem and the fixed-point theory applied to a positive cone on a Banach space, authors examine the positive coexistence of one prey and two competing predators in an interacting system with ratio-dependent functional responses under a hostile environment. Furthermore, Ko and Ahn [13, 14] investigate the stability at all non-negative equilibria and long time behavior of solutions for a ratio-dependent reaction-diffusion system. For more similar work, we refer the reader to [15-21].

On the other hand, one can find that an ecosystem in the real world is continuously distributed by some forces, which can result in changes in the biological parameters such as survival rates. Of practical interest in ecology is the question of whether or not an ecosystem can withstand those disturbances which persist for a finite period of time. In the language of control variables, we refer to the disturbance functions as control variables. This is of significance in the control of ecology balance. One of the methods for the realization of it is to alter the system structurally by introducing feedback control variables. The feedback control mechanism might be implemented by means of some biological control schemes or by harvesting procedure. In fact, during the last decade, the qualitative behavior of the population dynamics with feedback control has been studied extensively. Yin and Li [22] propose a single species model with feedback regulation and distributed time delay. By using the continuation theorem of coincidence degree theory, a criterion which guarantees the existence of a positive periodic solution of the system is obtained. Furthermore, Chen [23] obtains a sufficient condition which guarantees the global attractivity of the positive solution of the system by constructing a suitable Lyapunov functional. Nie et al. [24] consider a non-autonomous predator-prey Lotka-Volterra system with feedback controls. They study whether or not the feedback controls have an influence on the permanence of a positive solution of the general non-autonomous predator-prey LotkaVolterra type systems, and establish the general criteria on the permanence of the system, which is independent of some feedback controls. In additional, by constructing a suitable Lyapunov function, some sufficient conditions are obtained for the global stability of any positive solution to the system. More work on feedback controls can be found in [25-30].

Commonly, an ecological system, such as that represented by the deterministic LotkaVolterra model, is not suitable to describe the real behavior of the population dynamics. What we claim as "disturbance functions as control variables", which is mentioned above, is strictly connected to the environmental noise effect. It is necessary to include the effect of environmental variables that can be deterministic, such as the variation of the temperature due to atmospheric conditions, and stochastic, due to the stochastic variability of all the other variables, such as growth rate, resources, etc. [31-36]. Moreover, the study of nonlinear dynamical systems in the presence of external noise has led to the discovery of a number of counterintuitive phenomena, with a constructive role of the noise and high fundamental and practical interests in many scientific areas. The presence of a noise source can change the stability of the ecological system [37]. Ghergu and Radulescu [38] study the existence and non-existence of classical solutions to a general Gierer-Meinhardt 
system in which both the activator and the inhibitor have different sources given by general nonlinearities, and regularity and uniqueness of the solution in one dimension are also presented. In 2010, Ghergu and Radulescu [39] consider a class of reaction-diffusion system of Brusselator type, and show that if $f(u)$ has a sublinear growth then no Turing patterns occur, while if $f(u)$ has a superlinear growth then the existence of such patterns is strongly related to the inter-dependence between the parameters $a, b$ and the diffusion coefficients $d_{1}, d_{2}$. Liu et al. [40] establish the existence of at least four positive periodic solutions for a discrete time Lotka-Volterra competitive system with harvesting terms by using Mawhin's continuation theorem of coincidence degree theory. In [41], Giacomoni et al. investigate a quasilinear and singular elliptic system and give some applications to biology. In particular, many mathematical models in biology, chemistry, and population genetics are included and studied in [42]. In recent years many theoretical investigations have been done on noise-induced effects in population dynamics [43-45]. Finally, the noise source can be non-Gaussian and this further enriches the dynamics [46-51].

However, as far as we know, no work has been done for the one-predator and two-prey system with ratio-dependent functional responses and feedback controls. So, in this paper, we will consider the following system:

$$
\left\{\begin{array}{l}
\dot{x}_{1}(t)=x_{1}(t)\left[r_{1}(t)-a_{11}(t) x_{1}(t)-\frac{a_{12}(t) x_{2}(t)}{b_{12}(t) x_{2}(t)+x_{1}(t)}-\frac{a_{13}(t) x_{3}(t)}{b_{13}(t) x_{3}(t)+x_{1}(t)}-d_{1}(t) u_{1}(t)\right] \\
\dot{x}_{2}(t)=x_{2}(t)\left[-r_{2}(t)+\frac{a_{21}(t) x_{1}(t)}{b_{12}(t) x_{2}(t)+x_{1}(t)}-a_{23}(t) x_{3}(t)+d_{2}(t) u_{2}(t)\right] \\
\dot{x}_{3}(t)=x_{3}(t)\left[-r_{3}(t)+\frac{a_{31}(t) x_{1}(t)}{b_{13}(t) x_{3}(t)+x_{1}(t)}-a_{32}(t) x_{2}(t)+d_{3}(t) u_{3}(t)\right] \\
\dot{u}_{1}(t)=e_{1}(t)-f_{1}(t) u_{1}(t)+q_{1}(t) x_{1}(t) \\
\dot{u}_{2}(t)=e_{2}(t)-f_{2}(t) u_{2}(t)-q_{2}(t) x_{2}(t) \\
\dot{u}_{3}(t)=e_{3}(t)-f_{3}(t) u_{3}(t)-q_{3}(t) x_{3}(t)
\end{array}\right.
$$

where $x_{1}(t), x_{2}(t)$ and $x_{3}(t)$ stand for the densities of one prey and two competing predators, respectively, and $u_{i}(t)(i=1,2,3)$ are the indirect control variables. The given coefficients $a_{i j}(t), b_{i j}(t), d_{i}(t), e_{i}(t), f_{i}(t), q_{i}(t)$, and $r_{i}(t)$ are positive continuous bounded functions of $t$ for $i, j=1,2,3$. System (1.1) describes the interaction between prey and predator species which is based on a ratio-dependent functional response. System (1.1) is the socalled food web system with two competing predators and one prey. Here, $r_{1}(t)$ is the intrinsic growth rate in the absence of predators $r_{2}(t)$ and $x_{3}(t)$; the parameters $a_{12}(t)$ and $a_{13}(t)$ are the capturing (or catching efficiency) rates of the two predators; $a_{21}(t)$ and $a_{31}(t)$ are the conversion rates (or maximum growth rates); $b_{12}(t)$ and $b_{13}(t)$ are the interference coefficients of predator species; $r_{2}(t)$ and $r_{3}(t)$ are the death rates of the two predator species $x_{2}(t)$ and $x_{3}(t) ; d_{i}(t), e_{i}(t), q_{i}(t)$, and $f_{i}(t)(i=1,2,3)$ are the controls parameters. When there are no feedback controls, system (1.1) is reduced to the following Lotka and Volterra model:

$$
\left\{\begin{array}{l}
\dot{x}_{1}(t)=x_{1}(t)\left[a_{1}(t)-a_{11}(t) x_{1}(t)-\frac{a_{12}(t) x_{2}(t)}{b_{12}(t) x_{2}(t)+x_{1}(t)}-\frac{a_{13}(t) x_{3}(t)}{b_{13}(t) x_{3}(t)+x_{1}(t)}\right] \\
\dot{x}_{2}(t)=x_{2}(t)\left[-a_{2}(t)+\frac{a_{21}(t) x_{1}(t)}{b_{12}(t) x_{2}(t)+x_{1}(t)}-a_{23}(t) x_{3}(t)\right] \\
\dot{x}_{3}(t)=x_{3}(t)\left[-a_{3}(t)+\frac{a_{31}(t) x_{1}(t)}{b_{13}(t) x_{3}(t)+x_{1}(t)}-a_{32}(t) x_{2}(t)\right]
\end{array}\right.
$$

where the meanings of the parameters of system (1.2) are the same as those of (1.1). Lu et al. [52] show that this system is permanent and globally asymptotically stable under some 
appropriate conditions by constructing a suitable Lyapunov function. Comparing systems (1.1) and (1.2), one could see that we introduce the control variables $u_{i}(t)(i=1,2,3)$ so as to implement a feedback control mechanism.

This paper is organized as follows. In Sect. 2, we provide the conditions for permanence to system (1.1) by using a comparison theorem and developing some new analysis techniques. In Sect. 3, by constructing a non-negative Lyapunov function, we shall derive sufficient conditions for the global attractivity of positive solution for the predator-prey system (1.1). In Sect. 4, some conditions for the existence, uniqueness, and stability of a positive periodic solution for the corresponding periodic system are obtained by using the fixed point theory and some new analysis method. Some numerical solutions of the equations describing the system are given in Sect. 5 to verify that the obtained criteria are verifiable.

\section{Permanence}

In order to establish a permanence result for system (1.1), we need some preparations. Due to the biological interpretation of the system, it is reasonable to consider only positive solution of (1.1), in other words, to take admissible initial conditions $x_{i}\left(t_{0}\right)>0, u_{i}\left(t_{0}\right)>0$ $(i=1,2,3)$. Firstly, we introduce the following notations and definitions. Given a function $g(t)$ defined on $\left[t_{0},+\infty\right)$, we set

$$
g^{m}=\sup \left\{g(t): t_{0}<t<+\infty\right\}, \quad g^{l}=\inf \left\{g(t): t_{0}<t<+\infty\right\} .
$$

Definition 2.1 System (1.1) is called permanent if there exist positive constants $M_{i}, N_{i}$, $m_{i}, n_{i}(i=1,2,3)$, and $T$ such that $m_{i} \leq x_{i}(t) \leq M_{i}, n_{i} \leq u_{i}(t) \leq N_{i}$ as $t>T$ for any positive solution $\left(x_{1}(t), x_{2}(t), x_{3}(t), u_{1}(t), u_{2}(t), u_{3}(t)\right)$ of system (1.1) with positive initials.

For system (1.1), we let

$$
\begin{aligned}
& M_{1}^{*}=\frac{r_{1}^{m}}{a_{11}^{l}}, \quad N_{1}^{*}=\frac{e_{1}^{m}+q_{1}^{m} M_{1}}{f_{1}^{l}}, \\
& m_{1}^{*}=\frac{r_{1}^{l} b_{12}^{l} b_{13}^{l}-a_{12}^{m} b_{13}^{l}-a_{13}^{m} b_{13}^{l}-d_{1}^{m} b_{12}^{l} b_{13}^{l} N_{1}}{a_{11}^{m} b_{12}^{l} b_{13}^{l}}, \\
& N_{3}^{*}=\frac{e_{3}^{m}}{f_{3}^{l}}, \quad M_{3}^{*}=M_{1} \frac{d_{3}^{m} N_{3}+a_{31}^{m}-r_{3}^{l}}{r_{3}^{l} b_{13}^{l}-d_{3}^{m} b_{13}^{l} N_{3}} \\
& N_{2}^{*}=\frac{e_{2}^{m}}{f_{2}^{l}}, \quad M_{2}^{*}=M_{1} \frac{d_{2}^{m} N_{2}+a_{21}^{m}-r_{2}^{l}}{r_{2}^{l} b_{12}^{l}-d_{2}^{m} b_{12}^{l} N_{2}}, \\
& m_{2}^{*}=\frac{m_{1}\left(-a_{23}^{m} M_{3}+a_{21}^{l}-r_{2}^{m}\right)}{r_{2}^{m} b_{12}^{m}+a_{23}^{m} b_{12}^{m} M_{3}}, \quad m_{3}^{*}=\frac{m_{1}\left(-a_{23}^{m} M_{2}+a_{31}^{l}-r_{3}^{m}\right)}{r_{3}^{m} b_{13}^{m}+a_{32}^{m} b_{13}^{m} M_{2}}, \\
& n_{1}^{*}=\frac{e_{1}^{l}+q_{1}^{l} m_{1}}{f_{1}^{m}}, \quad n_{2}^{*}=\frac{e_{2}^{l}-q_{2}^{m} M_{2}}{f_{2}^{m}}, \quad n_{3}^{*}=\frac{e_{3}^{l}-q_{3}^{m} M_{3}}{f_{3}^{m}},
\end{aligned}
$$

where $M_{i}, N_{i}, m_{i}, n_{i}$ are some appropriate positive constants such that

$$
0<m_{i}<m_{i}^{*}<M_{i}^{*}<M_{i}, \quad 0<n_{i}<n_{i}^{*}<N_{i}^{*}<N_{i}, \quad i=1,2,3 .
$$

Theorem 2.1 Assume that system (1.1) satisfies the following conditions: 
$\left(\mathrm{H}_{1}\right)$

$$
r_{1}^{l} b_{12}^{l} b_{13}^{l}>a_{12}^{m} b_{13}^{l}+a_{13}^{m} b_{13}^{l}+d_{1}^{m} b_{12}^{l} b_{13}^{l} N_{1}
$$

$\left(\mathrm{H}_{2}\right)$

$$
\frac{d_{3}^{m} N_{3}+a_{31}^{m}-r_{3}^{l}}{r_{3}^{l} b_{13}^{l}-d_{3}^{m} b_{13}^{l} N_{3}}>0
$$

$\left(\mathrm{H}_{3}\right)$

$$
\frac{d_{2}^{m} N_{2}+a_{21}^{m}-r_{2}^{l}}{r_{2}^{l} b_{12}^{l}-d_{2}^{m} b_{12}^{l} N_{2}}>0
$$

$\left(\mathrm{H}_{4}\right)$

$$
-a_{23}^{m} M_{3}+a_{21}^{l}>r_{2}^{m}
$$

$\left(\mathrm{H}_{5}\right)$

$$
-a_{23}^{m} M_{2}+a_{31}^{l}>r_{3}^{m}
$$

$\left(\mathrm{H}_{6}\right)$

$$
e_{2}^{l}>q_{2}^{m} M_{2}
$$

$\left(\mathrm{H}_{7}\right)$

$$
e_{3}^{l}>q_{3}^{m} M_{3}
$$

Then system (1.1) is permanent.

Proof From the first equation of system (1.1), we have

$$
\dot{x}_{1}(t) \leq x_{1}(t)\left[r_{1}(t)-a_{11}(t) x_{1}(t)\right] \leq x_{1}(t)\left[r_{1}^{m}-a_{11}^{l} x_{1}(t)\right]=a_{11}^{l} x_{1}(t)\left[M_{1}^{*}-x_{1}(t)\right],
$$

thus, it holds that

$$
\left.\dot{x}_{1}(t)\right|_{x_{1}(t)=M_{i}^{*}} \leq a_{11}^{l} M_{1}\left[M_{1}^{*}-M_{1}\right]<0 .
$$

In view of the comparison theorem, one has

(1) When $0<x_{1}\left(t_{0}\right)<M_{1}$, if $t \geq t_{0}$, then $x_{1}(t) \leq M_{1}$.

(2) When $x_{1}\left(t_{0}\right) \geq M_{1}$, for a sufficiently large $t$, one has $x_{1}(t) \leq M_{1}$. Otherwise, if $x_{1}(t)>M_{1}$, then there exists $\alpha>0$ such that $x_{1}(t) \geq M_{1}^{*}+\alpha$. Moreover, we have

$$
\left.\dot{x}_{1}(t)\right|_{x_{1}(t)>M_{1}} \leq x_{1}(t)\left[r_{1}(t)-a_{11}(t) x_{1}(t)\right] \leq a_{11}^{l} x_{1}(t)\left[M_{1}^{*}-x_{1}(t)\right]<-a_{11}^{l} \alpha x_{1}(t),
$$


thus, it holds that $x_{1}(t)<x_{1}\left(t_{0}\right) \exp \left(-a_{11}^{l} \alpha t\right) \rightarrow 0$ as $t \rightarrow+\infty$. This is a contradiction, so there exist sufficiently large $T_{1} \geq t_{0} \geq 0$ such that

$$
x_{1}(t) \leq M_{1} \quad \text { as } t>T_{1} \text {. }
$$

By the sixth equation of system (1.1), we can get

$$
\dot{u}_{3} \leq e_{3}^{m}-f_{3}^{l} u_{3}(t)=f_{3}^{l}\left[N_{3}^{*}-u_{3}(t)\right]
$$

Thus, employing the comparison theorem and analysis similar to the one used above, it holds that there exist sufficiently large $T_{6} \geq t_{0} \geq 0$ such that

$$
u_{3}(t) \leq N_{3} \quad \text { as } t>T_{6}
$$

Similarly, from the fifth equation of model (1.1), one has that there exist sufficiently large $T_{5} \geq t_{0} \geq 0$ such that

$$
u_{2}(t) \leq N_{2} \quad \text { as } t>T_{5}
$$

From the third equation of system (1.1), and combining (2.1) and (2.2), the following holds:

$$
\begin{aligned}
\dot{x}_{3} & \leq x_{3}(t)\left[-r_{3}^{l}+\frac{a_{31}^{m} M_{1}}{b_{13}(t) x_{3}(t)+M_{1}}+d_{3}^{m} N_{3}\right] \\
& \leq x_{3}(t)\left[-r_{3}^{l}+\frac{a_{31}^{m} M_{1}}{b_{13}^{l} x_{3}(t)+M_{1}}+d_{3}^{m} N_{3}\right] \\
& =x_{3}(t)\left[\frac{-r_{3}^{l}\left(b_{13}^{l} x_{3}(t)+M_{1}\right)+a_{31}^{m} M_{1}+d_{3}^{m} N_{3}\left(b_{13}^{l} x_{3}(t)+M_{1}\right)}{b_{13}^{l} x_{3}(t)+M_{1}}\right] \\
& =x_{3}(t) \frac{r_{3}^{l} b_{13}^{l}-d_{3}^{m} N_{3} b_{13}^{l}}{b_{13}^{l} x_{3}(t)+M_{1}}\left[-x_{3}(t)+\frac{M_{1}\left(d_{3}^{m} N_{3}+a_{31}^{m}-r_{3}^{l}\right)}{r_{3}^{l} b_{13}^{l}-d_{3}^{m} N_{3} b_{13}^{l}}\right] \\
& =x_{3}(t) \frac{r_{3}^{l} b_{13}^{l}-d_{3}^{m} N_{3} b_{13}^{l}}{b_{13}^{l} x_{3}(t)+M_{1}}\left[-x_{3}(t)+M_{3}\right] .
\end{aligned}
$$

In view of the comparison theorem and the same analysis as above, we can obtain that

(3) When $0<x_{3}\left(t_{0}\right)<M_{3}$, if $t \geq t_{0}$, then $x_{3}(t) \leq M_{3}$;

(4) When $x_{3}\left(t_{0}\right) \geq M_{3}$, for a sufficiently large $t$, one has $x_{3}(t) \leq M_{3}$. So it holds that there exist sufficiently large $T_{3} \geq t_{0} \geq 0$ such that

$$
x_{3}(t) \leq M_{3} \quad \text { as } t>T_{3} \text {. }
$$

Similarly, from the second equation of system (1.1), and combining (2.1) and (2.3), it holds that there exist sufficiently large $T_{3} \geq t_{0} \geq 0$ such that

$$
x_{2}(t) \leq M_{2} \quad \text { as } t>T_{2} \text {. }
$$


By the fourth equation of system (1.1), we can obtain

$$
\dot{u}_{1} \leq e_{1}^{m}-f_{1}^{l} u_{1}(t)+q_{1}^{m} M_{1}=f_{1}^{l}\left[N_{1}^{*}-u_{1}(t)\right]
$$

Moreover, employing the comparison theorem and analysis similar to the one used above, it holds that there exist sufficiently large $T_{4} \geq t_{0} \geq 0$ such that

$$
u_{1}(t) \leq N_{1} \quad \text { as } t>T_{4}
$$

On the other hand, by the same analysis process, we have

$$
\begin{aligned}
\dot{x}_{1}(t) & \geq x_{1}(t)\left[r_{1}^{l}-a_{11}^{m} x_{1}(t)-\frac{a_{12}^{m}}{b_{12}^{l}}-\frac{a_{13}^{m}}{b_{13}^{l}}-d_{1}^{m} N_{1}\right] \\
& =x_{1}(t) a_{11}^{m}\left[-x_{1}(t)+\frac{r_{1}^{l} b_{12}^{l} b_{13}^{l}-a_{12}^{m} b_{13}^{l}-a_{13}^{m} b_{12}^{l}-b_{12}^{l} b_{13}^{l} d_{1}^{m} N_{1}}{a_{11}^{m} b_{12}^{l} b_{13}^{l}}\right] \\
& =x_{1}(t) a_{11}^{m}\left[-x_{1}(t)+m_{1}^{*}\right] .
\end{aligned}
$$

Using the comparison theorem and the same analysis as above, it holds that

(5) When $m_{1}<x_{1}\left(t_{0}\right)$, if $t \geq t_{0}$, then $m_{1} \leq x_{1}(t)$;

(6) When $0<x_{1}\left(t_{0}\right) \leq m_{1}$, for a sufficiently large $t$, one has $m_{1} \leq x_{1}(t)$. Otherwise, if $x_{1}(t)<m_{1}$, then there exists $\beta>0$ such that $x_{1}(t) \leq m_{1}^{*}-\beta$. Moreover, we have

$$
\left.\dot{x}_{1}(t)\right|_{x_{1}(t)<m_{1}} \geq a_{11}^{m} x_{1}(t)\left[-x_{1}(t)+m_{1}^{*}\right]>a_{11}^{m} \beta x_{1}(t),
$$

thus, it holds that $x_{1}(t)>x_{1}\left(t_{0}\right) \exp \left(a_{11}^{m} \beta t\right) \rightarrow+\infty$ as $t \rightarrow+\infty$. This is a contradiction, so there exist sufficiently large $T_{1}^{\prime} \geq t_{0} \geq 0$ such that

$$
x_{1}(t) \geq m_{1} \quad \text { as } t>T_{1}^{\prime}
$$

Similarly, we can obtain that there exist five sufficiently large positive constants $T_{i}^{\prime}$ $(i=2, \ldots, 6)$ such that

$$
x_{i}(t) \geq m_{i}, \quad \text { as } t>T_{i}^{\prime}, i=2, \ldots, 6 .
$$

From (2.1)-(2.8), and setting $T=\max _{1 \leq i \leq 6}\left\{T_{i}, T_{i}^{\prime}\right\}$, we have $m_{i} \leq x_{i}(t) \leq M_{i}, n_{i} \leq u_{i}(t) \leq N_{i}$ as $t>T$ for any positive solution $\left(x_{1}(t), x_{2}(t), x_{3}(t), u_{1}(t), u_{2}(t), u_{3}(t)\right)$ of system (1.1) with positive initials. This ends the proof of Theorem 2.1 .

\section{Global attractivity}

In this section, the global attractivity of system (1.1) is studied. To get the sufficient conditions for global attractivity of system (1.1), the following definition and lemma are firstly given.

Definition 3.1 System (1.1) is said to be globally attractive if there exists a positive solution $X(t)=\left(x_{1}(t), x_{2}(t), x_{3}(t), u_{1}(t), u_{2}(x), u_{3}(x)\right)$ of system $(1.1)$ such that

$$
\lim _{t \rightarrow+\infty}\left|x_{i}(t)-y_{i}(t)\right|=0, \quad \lim _{t \rightarrow+\infty}\left|u_{i}(t)-v_{i}(t)\right|=0
$$


for any other positive solution $Y(t)=\left(y_{1}(t), y_{2}(t), y_{3}(t), v_{1}(t), v_{2}(t), v_{3}(t)\right)$ of system (1.1).

Lemma 3.1 ([53]) If the function $f(t): R^{+} \rightarrow R$ is uniformly continuous, and the limit $\lim _{t \rightarrow \infty} \int_{0}^{t} f(s) d s$ exists and is finite, then $\lim _{t \rightarrow+\infty} f(t)=0$.

Theorem 3.1 Assume that system (1.1) satisfies $\left(\mathrm{H}_{1}\right)-\left(\mathrm{H}_{7}\right)$ and the following conditions:

$\left(\mathrm{H}_{8}\right)$

$$
a_{11}^{l}-\frac{\left(a_{12}^{m}+b_{12}^{m} a_{21}^{m}\right) M_{2}}{\left(b_{12}^{l} m_{2}+m_{1}\right)^{2}}-\frac{\left(a_{13}^{m}+b_{13}^{m} a_{31}^{m}\right) M_{3}}{\left(b_{13}^{l} m_{3}+m_{1}\right)^{2}}-q_{1}^{m}>0
$$

$\left(\mathrm{H}_{9}\right)$

$$
-a_{32}^{m}-\frac{a_{12}^{m} M_{1}}{\left(b_{12}^{l} m_{2}+m_{1}\right)^{2}}+\frac{a_{21}^{l} b_{12}^{l} m_{1}}{\left(b_{12}^{m} M_{2}+M_{1}\right)^{2}}-q_{2}^{m}>0
$$

$\left(\mathrm{H}_{10}\right)$

$$
-a_{23}^{m}-\frac{a_{13}^{m} M_{1}}{\left(b_{13}^{l} m_{3}+m_{1}\right)^{2}}+\frac{a_{31}^{l} b_{13}^{l} m_{1}}{\left(b_{13}^{m} M_{3}+M_{1}\right)^{2}}-q_{3}^{m}>0
$$

$\left(\mathrm{H}_{11}\right)$

$$
f_{i}^{l}>d_{i}^{m} \quad(i=1,2,3)
$$

Then system (1.1) is globally attractive.

Proof Let $X(t)=\left(x_{1}(t), x_{2}(t), x_{3}(t), u_{1}(t), u_{2}(t), u_{3}(t)\right)$ be a positive solution of system (1.1) and $Y(t)=\left(y_{1}(t), y_{2}(t), y_{3}(t), v_{1}(t), v_{2}(t), v_{3}(t)\right)$ be any positive solution of system (1.1) with initial conditions $x_{i}\left(t_{0}\right)>0, u_{i}\left(t_{0}\right)>0, i=1,2,3$, then from Theorem 2.1, there exist positive constants $M_{i}, N_{i}, m_{i}, n_{i}$, and $T$ such that $m_{i} \leq x_{i}(t) \leq M_{i}, n_{i} \leq u_{i}(t) \leq N_{i}$ for all $t>T$.

Set the Lyapunov function

$$
V(t)=\sum_{i=1}^{3}\left[\left|\ln x_{i}(t)-\ln y_{i}(t)\right|+\left|u_{i}(t)-v_{i}(t)\right|\right] .
$$

We compute the upper right derivative of $V(t)$ along with the solution of system (1.1) only using $\mathrm{H}$-assumptions and simple calculations:

$$
\begin{aligned}
D^{+} V(t) \leq & \sum_{i=1}^{3} D^{+}\left[\left|\ln x_{i}(t)-\ln y_{i}(t)\right|+\left|u_{i}(t)-v_{i}(t)\right|\right] \\
= & \sum_{i=1}^{3} \operatorname{sgn}\left\{x_{i}(t)-y_{i}(t)\right\}\left(\frac{\dot{x}_{i}(t)}{x_{i}(t)}-\frac{\dot{y}_{i}(t)}{y_{i}(t)}\right)+\sum_{i=1}^{3} \operatorname{sgn}\left\{u_{i}(t)-v_{i}(t)\right\}\left(\dot{u}_{i}(t)-\dot{v}_{i}(t)\right) \\
= & \operatorname{sgn}\left\{x_{1}(t)-y_{1}(t)\right\}\left[-a_{11}(t)\left(x_{1}(t)-y_{1}(t)\right)-a_{12}(t)\left(\frac{x_{2}(t)}{\left(b_{12}(t) x_{2}(t)+x_{1}(t)\right)}\right.\right. \\
& \left.-\frac{y_{2}(t)}{\left(b_{12}(t) y_{2}(t)+y_{1}(t)\right)}\right)
\end{aligned}
$$




$$
\begin{aligned}
& -a_{13}(t)\left(\frac{x_{3}(t)}{\left(b_{13}(t) x_{3}(t)+x_{1}(t)\right)}-\frac{y_{3}(t)}{\left(b_{13}(t) y_{3}(t)+y_{1}(t)\right)}\right) \\
& \left.-d_{1}(t)\left(u_{1}(t)-v_{1}(t)\right)\right]+\operatorname{sgn}\left\{x_{2}(t)-y_{2}(t)\right\}\left[-a_{23}(t)\left(x_{3}(t)-y_{3}(t)\right)\right. \\
& \left.+a_{21}(t)\left(\frac{x_{1}(t)}{b_{12}(t) x_{2}(t)+x_{1}(t)}-\frac{y_{1}(t)}{b_{12}(t) y_{2}(t)+y_{1}(t)}\right)+d_{2}(t)\left(u_{2}(t)-v_{2}(t)\right)\right] \\
& +\operatorname{sgn}\left\{x_{3}(t)-y_{3}(t)\right\} \\
& \times\left[-a_{32}\left(x_{2}(t)-y_{2}(t)\right)+a_{31}(t)\left(\frac{x_{1}(t)}{b_{13}(t) x_{3}(t)+x_{1}(t)}-\frac{y_{1}(t)}{b_{13}(t) y_{3}(t)+y_{1}(t)}\right)\right. \\
& \left.+d_{3}(t)\left(u_{3}(t)-v_{3}(t)\right)\right] \\
& +\operatorname{sgn}\left\{u_{1}(t)-v_{1}(t)\right\}\left[-f_{1}(t)\left(u_{1}(t)-v_{1}(t)\right)+q_{1}(t)\left(x_{1}(t)-y_{1}(t)\right)\right] \\
& +\operatorname{sgn}\left\{u_{2}(t)-v_{2}(t)\right\}\left[-f_{2}(t)\left(u_{2}(t)-v_{2}(t)\right)-q_{2}(t)\left(x_{2}(t)-y_{2}(t)\right)\right] \\
& +\operatorname{sgn}\left\{u_{3}(t)-v_{3}(t)\right\}\left[-f_{3}(t)\left(u_{3}(t)-v_{3}(t)\right)-q_{3}(t)\left(x_{3}(t)-y_{3}(t)\right)\right] \\
& =\operatorname{sgn}\left\{x_{1}(t)-y_{1}(t)\right\}\left[-a_{11}(t)\left(x_{1}(t)-y_{1}(t)\right)\right. \\
& -a_{12}(t)\left(\frac{y_{1}(t)\left(x_{2}(t)-y_{2}(t)\right)-y_{2}(t)\left(x_{1}(t)-y_{1}(t)\right)}{\left(b_{12}(t) x_{2}(t)+x_{1}(t)\right)\left(b_{12}(t) y_{2}(t)+y_{1}(t)\right.}\right) \\
& \left.-a_{13}(t)\left(\frac{y_{1}(t)\left(x_{3}(t)-y_{3}(t)\right)-y_{3}(t)\left(x_{1}(t)-y_{1}(t)\right)}{\left(b_{13}(t) x_{3}(t)+x_{1}(t)\right)\left(b_{13}(t) y_{3}(t)+x_{1}(t)\right)}\right)-d_{1}(t)\left(u_{1}(t)-v_{1}(t)\right)\right] \\
& +\operatorname{sgn}\left\{x_{2}(t)-y_{2}(t)\right\}\left[a_{21}(t) b_{12}(t)\left(\frac{y_{2}(t)\left(x_{1}(t)-y_{1}(t)\right)-y_{1}(t)\left(x_{2}(t)-y_{2}(t)\right)}{\left(b_{12}(t) x_{2}(t)+x_{1}(t)\right)\left(b_{12}(t) y_{2}(t)+y_{1}(t)\right.}\right)\right. \\
& \left.-a_{23}(t)\left(x_{3}(t)-y_{3}(t)\right)+d_{2}(t)\left(u_{2}(t)-v_{2}(t)\right)\right] \\
& +\operatorname{sgn}\left\{x_{3}(t)-y_{3}(t)\right\}\left[a_{31}(t) b_{13}(t)\left(\frac{y_{3}(t)\left(x_{1}(t)-y_{1}(t)\right)-y_{1}(t)\left(x_{3}(t)-y_{3}(t)\right)}{\left(b_{13}(t) x_{3}(t)+x_{1}(t)\right)\left(b_{13}(t) y_{3}(t)+y_{1}(t)\right.}\right)\right. \\
& \left.-a_{23}(t)\left(x_{2}(t)-y_{2}(t)\right)+d_{3}(t)\left(u_{3}(t)-v_{3}(t)\right)\right] \\
& +\operatorname{sgn}\left\{u_{1}(t)-v_{1}(t)\right\}\left[-f_{1}(t)\left(u_{1}(t)-v_{1}(t)\right)+q_{1}(t)\left(x_{1}(t)-y_{1}(t)\right)\right] \\
& +\operatorname{sgn}\left\{u_{2}(t)-v_{2}(t)\right\}\left[-f_{2}(t)\left(u_{2}(t)-v_{2}(t)\right)-q_{2}(t)\left(x_{2}(t)-y_{2}(t)\right)\right] \\
& +\operatorname{sgn}\left\{u_{3}(t)-v_{3}(t)\right\}\left[-f_{3}(t)\left(u_{3}(t)-v_{3}(t)\right)-q_{3}(t)\left(x_{3}(t)-y_{3}(t)\right)\right] \\
& \leq\left|x_{1}(t)-y_{1}(t)\right|\left[-a_{11}(t)+\frac{\left(a_{12}(t)+b_{12}(t) a_{21}(t)\right) y_{2}(t)}{\left(b_{12}(t) x_{2}(t)+x_{1}(t)\right)\left(b_{12}(t) y_{2}(t)+y_{1}(t)\right)}\right. \\
& \left.+\frac{\left(a_{13}(t)+b_{13}(t) a_{31}(t)\right) y_{3}(t)}{\left(b_{13}(t) x_{3}(t)+x_{1}(t)\right)\left(b_{13}(t) y_{3}(t)+y_{1}(t)\right)}+q_{1}(t)\right] \\
& +\left|x_{2}(t)-y_{2}(t)\right|\left[a_{32}(t)+\frac{\left(a_{12}(t)-b_{12}(t) a_{21}(t)\right) y_{1}(t)}{\left(b_{12}(t) x_{2}(t)+x_{1}(t)\right)\left(b_{12}(t) y_{2}(t)+y_{1}(t)\right)}+q_{2}(t)\right] \\
& +\left|x_{3}(t)-y_{3}(t)\right|\left[a_{23}(t)+\frac{\left(a_{13}(t)-b_{13}(t) a_{31}(t)\right) y_{1}(t)}{\left(b_{13}(t) x_{2}(t)+x_{1}(t)\right)\left(b_{13}(t) y_{2}(t)+y_{1}(t)\right)}+q_{3}(t)\right] \\
& +\left|u_{1}(t)-v_{1}(t)\right|\left[-f_{1}(t)+d_{1}(t)\right]+\left|u_{2}(t)-v_{2}(t)\right|\left[-f_{2}(t)+d_{2}(t)\right]
\end{aligned}
$$




$$
\begin{aligned}
& +\left|u_{3}(t)-v_{3}(t)\right|\left[-f_{3}(t)+d_{3}(t)\right] \\
\leq & -\left|x_{1}(t)-y_{1}(t)\right|\left[a_{11}^{l}-\frac{\left(a_{12}^{m}+b_{12}^{m} a_{21}^{m}\right) M_{2}}{\left(b_{12}^{l} m_{2}+m_{1}\right)^{2}}-\frac{\left(a_{13}^{m}+b_{13}^{m} a_{31}^{m}\right) M_{3}}{\left(b_{13}^{l} m_{3}+m_{1}\right)^{2}}-q_{1}^{m}\right] \\
& -\left|x_{2}(t)-y_{2}(t)\right|\left[-a_{32}^{m}-\frac{a_{12}^{m} M_{1}}{\left(b_{12}^{l} m_{2}+m_{1}\right)^{2}}+\frac{a_{21}^{l} b_{12}^{l} m_{1}}{\left(b_{12}^{m} M_{2}+M_{1}\right)^{2}}-q_{2}^{m}\right] \\
& -\left|x_{3}(t)-y_{3}(t)\right|\left[-a_{23}^{m}-\frac{a_{13}^{m} M_{1}}{\left(b_{13}^{l} m_{3}+m_{1}\right)^{2}}+\frac{a_{31}^{l} b_{13}^{l} m_{1}}{\left(b_{13}^{m} M_{3}+M_{1}\right)^{2}}-q_{3}^{m}\right] \\
& -\left|u_{1}(t)-v_{1}(t)\right|\left[f_{1}^{l}-d_{1}^{m}\right]-\left|u_{2}(t)-v_{2}(t)\right|\left[f_{2}^{l}-d_{2}^{m}\right] \\
& -\left|u_{3}(t)-v_{3}(t)\right|\left[f_{3}^{l}-d_{3}^{m}\right] .
\end{aligned}
$$

In view of conditions $\left(\mathrm{H}_{8}\right)-\left(\mathrm{H}_{11}\right)$, one has

$$
\begin{aligned}
\alpha= & \min \left\{a_{11}^{l}-\frac{\left(a_{12}^{m}+b_{12}^{m} a_{21}^{m}\right) M_{2}}{\left(b_{12}^{l} m_{2}+m_{1}\right)^{2}}-\frac{\left(a_{13}^{m}+b_{13}^{m} a_{31}^{m}\right) M_{3}}{\left(b_{13}^{l} m_{3}+m_{1}\right)^{2}}-q_{1}^{m},\right. \\
& -a_{32}^{m}-\frac{a_{12}^{m} M_{1}}{\left(b_{12}^{l} m_{2}+m_{1}\right)^{2}}+\frac{a_{21}^{l} b_{12}^{l} m_{1}}{\left(b_{12}^{m} M_{2}+M_{1}\right)^{2}}-q_{2}^{m}, \\
& \left.-a_{23}^{m}-\frac{a_{13}^{m} M_{1}}{\left(b_{13}^{l} m_{3}+m_{1}\right)^{2}}+\frac{a_{31}^{l} b_{13}^{l} m_{1}}{\left(b_{13}^{m} M_{3}+M_{1}\right)^{2}}-q_{3}^{m}, f_{1}^{l}-d_{1}^{m}, f_{2}^{l}-d_{2}^{m}, f_{3}^{l}-d_{3}^{m}\right\}>0 .
\end{aligned}
$$

Thus,

$$
D^{+} V(t) \leq-\alpha \sum_{i=1}^{3}\left[\left|x_{i}(t)-y_{i}(t)\right|+\left|u_{i}(t)-v_{i}(t)\right|\right]
$$

Integrating (3.1) from $T$ to $t\left(T \geq t_{0}\right)$, one has

$$
V(t)+\alpha \int_{T}^{t}\left\{\sum_{i=1}^{3}\left[\left|x_{i}(t)-y_{i}(t)\right|+\left|u_{i}(t)-v_{i}(t)\right|\right]\right\} d s \leq V(T)<+\infty .
$$

Therefore,

$$
\int_{T}^{t}\left\{\sum_{i=1}^{3}\left[\left|x_{i}(t)-y_{i}(t)\right|+\left|u_{i}(t)-v_{i}(t)\right|\right]\right\} d s \leq \frac{V(T)}{\alpha}<+\infty .
$$

By (3.2), we have

$$
\sum_{i=1}^{3}\left[\left|x_{i}(t)-y_{i}(t)\right|+\left|u_{i}(t)-v_{i}(t)\right|\right] \in L^{1}(T,+\infty)
$$

From the uniform permanence of system (1.1), $\sum_{i=1}^{3}\left[\left|x_{i}(t)-y_{i}(t)\right|+\left|u_{i}(t)-v_{i}(t)\right|\right]$ is uniformly continuous. By Lemma 3.1, we can obtain that

$$
\lim _{t \rightarrow+\infty}\left|x_{i}(t)-y_{i}(t)\right|=0, \quad \lim _{t \rightarrow+\infty}\left|u_{i}(t)-v_{i}(t)\right|=0 \quad(i=1,2,3) .
$$

This ends the proof of Theorem 3.1. 


\section{Periodic solution}

Assuming that the coefficients of system (1.1) are positive continuous, $\omega$-periodic functions, then system (1.1) is changed to an $\omega$-periodic system. In this section, we shall obtain conditions for the existence, uniqueness, and stability of a positive periodic solution for system (1.1) by using the fixed point theory and some new analysis method. For convenience, we give firstly the following lemma.

Lemma 4.1 ([54]) Let $S \subset R_{n}$ be convex and compact. If the mapping $T: S \rightarrow S$ is continuous, then there exists a fixed point, i.e., there exists $x^{*} \in S$ such that $T\left(x^{*}\right)=x^{*}$.

Theorem 4.1 Assume that system (1.1) is an w-periodic system and satisfies conditions $\left(\mathrm{H}_{1}\right)-\left(\mathrm{H}_{11}\right)$, then system $(1.1)$ has a positive unique $\omega$-periodic solution, which is globally asymptotically stable.

Proof According to the existence and uniqueness theorem of solutions of differential equations, we can obtain a Poincaré mapping $T: R_{+}^{6} \rightarrow R_{+}^{6}$ defined as follows:

$$
T\left(X_{0}\right)=X\left(t, \omega, t_{0}, X_{0}\right)
$$

where $X\left(t, \omega, t_{0}, X_{0}\right)=\left(x_{1}(t), x_{2}(t), x_{3}(t), u_{1}(t), u_{2}(t), u_{3}(t)\right)$ is a positive solution of system (1.1) with the initial conditions $X_{0}=\left(x_{1}\left(t_{0}\right), x_{2}\left(t_{0}\right), x_{3}\left(t_{0}\right), u_{1}\left(t_{0}\right), u_{2}\left(t_{0}\right), u_{3}\left(t_{0}\right)\right)$. And define

$$
S=\left\{\left(x_{1}, x_{2}, x_{3}, u_{1}, u_{2}, u_{3}\right) \in R_{+}^{6} \mid m_{i} \leq x_{i} \leq M_{i}, n_{i} \leq u_{i} \leq N_{i}, i=1,2,3\right\},
$$

then it is obvious that $S \subset R_{+}^{6}$ is a convex and compact set. By Theorem 2.1 and the continuity of solution of system (1.1) with respect to the initial conditions, the mapping $T: S \rightarrow S$ is continuous. Furthermore, it is not difficult to show that system (1.1) has a positive unique $\omega$-periodic solution, which is globally asymptotically stable by using Lemma 4.1, Theorem 2.1, and Theorem 3.1.

\section{Numerical simulation}

In this section, we give some numerical simulations supporting our theoretical analysis. As an example, we consider the following Lotka-Volterra predator-prey system with ratiodependent functional responses and feedback controls:

$$
\left\{\begin{aligned}
\dot{x}_{1}(t)= & x_{1}(t)\left[5+\cos \pi t-[6.25+1.25 \sin \pi t] x_{1}(t)-[0.4+0.1 \sin \pi t] x_{2}(t)\right. \\
& \left.-\frac{[0.75+0.25 \sin \pi t] x_{3}(t)}{[1.5+0.5 \sin \pi t] x_{3}(t)+x_{1}(t)}-(0.015+0.005 \sin \pi t) u_{1}(t)\right], \\
\dot{x}_{2}(t)= & x_{2}(t)\left[2.5+0.5 \cos \pi t-[0.13+0.1 \sin \pi t] x_{1}(t)-[7+\sin \pi t] x_{2}(t)\right. \\
& \left.-\frac{[1+0.5 \sin \pi t] x_{3}(t)}{[1.8+0.2 \sin \pi t] x_{3}(t)+x_{2}(t)}-(0.5+0.1 \sin \pi t) u_{2}(t)\right], \\
\dot{x_{3}}(t)= & x_{3}(t)\left[-[4.25+0.25 \cos \pi t]+\frac{[7.5+0.5 \sin \pi t] x_{1}(t)}{[1.5+0.5 \sin \pi t] x_{3}(t)+x_{1}(t)}\right. \\
& \left.+\frac{[5.5+0.5 \sin \pi t] x_{2}(t)}{[1.8+0.2 \sin \pi t] x_{3}(t)+x_{2}(t)}+(0.03+0.01 \sin \pi t) u_{3}(t)\right], \\
\dot{u}_{1}(t)= & {[0.8+0.2 \cos \pi t]-[1.6+0.1 \sin \pi t] u_{1}(t)+[0.15+0.05 \sin \pi t] x_{1}(t), } \\
\dot{u}_{2}(t)= & {[0.5+0.1 \cos \pi t]-[1.4+0.2 \sin \pi t] u_{2}(t)+[0.30+0.1 \sin \pi t] x_{2}(t), } \\
\dot{u}_{3}(t)= & {[2+0.5 \cos \pi t]-[1.2+0.2 \sin \pi t] u_{3}(t)-[0.15+0.01 \sin \pi t] x_{3}(t) . }
\end{aligned}\right.
$$




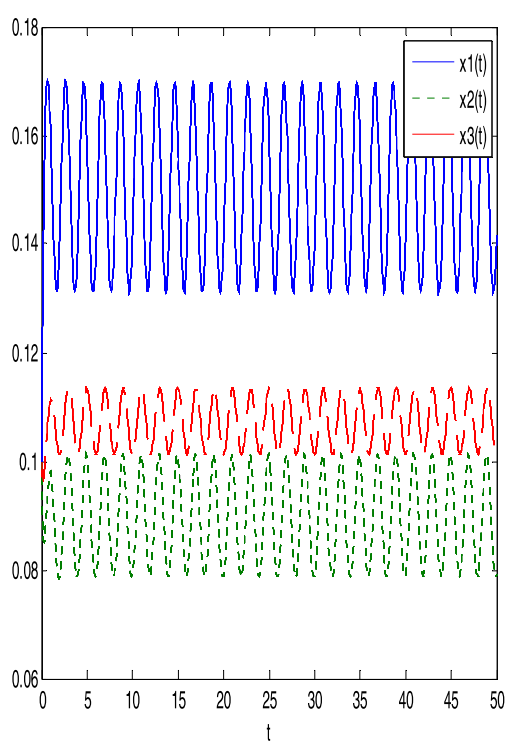

(a)

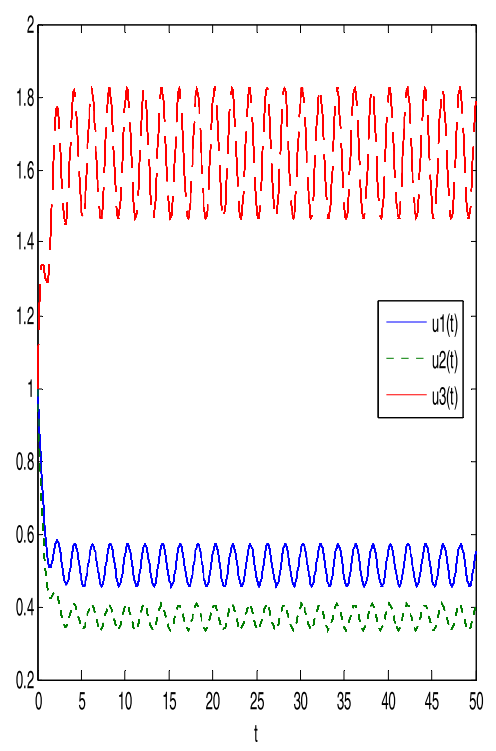

(b)

Figure 1 The numerical solution of system (5.1) with initial conditions (5.2). (a) Time series of $x_{1}(t), x_{2}(t)$, and $x_{3}(t)$. (b) Time series of $u_{1}(t), u_{2}(t)$, and $u_{3}(t)$

It is easy to show that system (5.1) satisfies the conditions of Theorem 4.1. It follows from Theorem 2.1, Theorem 3.1, and Theorem 4.1 that the predator-prey system (5.1) is permanent and globally attractive and that it has a unique and stable periodic solution which is globally asymptotically stable. By employing the software package MATLAB 7.1, we can solve the numerical solutions of Eqs. (5.1) which are shown in Fig. 1, Fig. 2, and Fig. 3. Figure 1 shows the permanence of system (5.1) with the initial conditions

$$
\begin{aligned}
& x_{1}\left(t_{0}\right)=0.1, \quad x_{2}\left(t_{0}\right)=0.1, \quad x_{3}\left(t_{0}\right)=0.1, \\
& u_{1}\left(t_{0}\right)=1, \quad u_{2}\left(t_{0}\right)=1, \quad u_{3}\left(t_{0}\right)=1 .
\end{aligned}
$$

From Fig. 1, it is not difficult to find that

$$
0.07 \leq x_{i}(t) \leq 0.18, \quad i=1,2,3,
$$

and

$$
0.2 \leq u_{i}(t) \leq 2, \quad i=1,2,3 \text { as } t>10 .
$$

From Fig. 2, one can find that $\lim _{t \rightarrow+\infty}\left|x_{i}(t)-y_{i}(t)\right|=0, i=1,2,3$, for any two solutions $X(t)=\left(x_{1}(t), x_{2}(t), x_{3}(t)\right)$ and $Y(t)=\left(y_{1}(t), y_{2}(t), y_{3}(t)\right)$ of system (5.1) with different initial conditions, which shows that the periodic predator-prey system (5.1) has a unique periodic solution which is globally asymptotically stable. Figure 3 shows the dynamic behavior of system (5.1). 


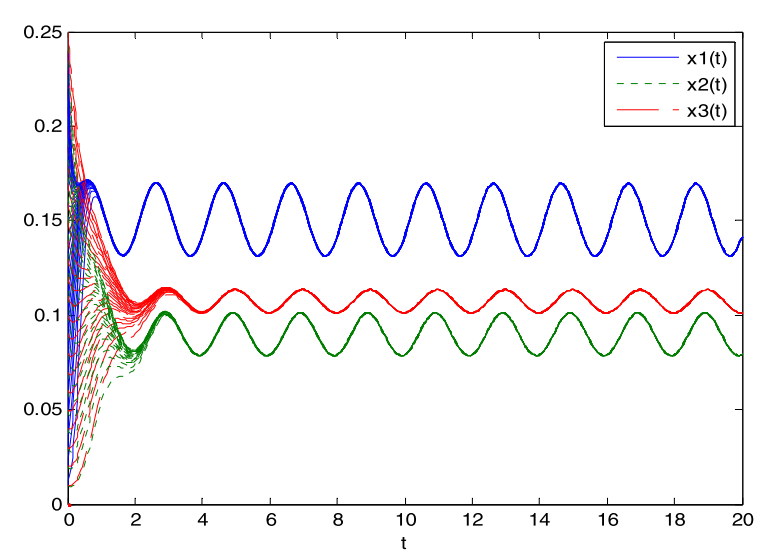

Figure 2 The numerical solution of system (5.1) with different initial conditions

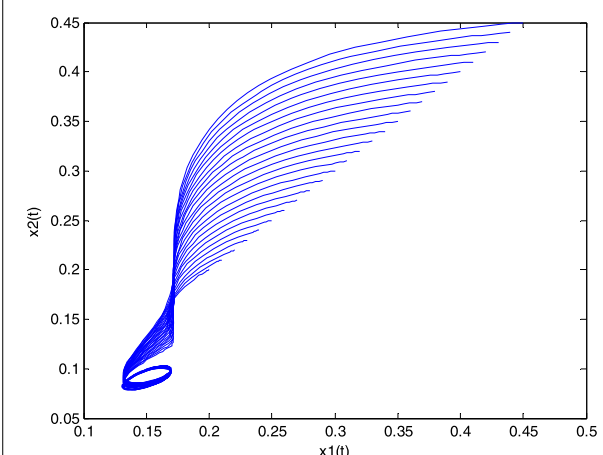

(a)

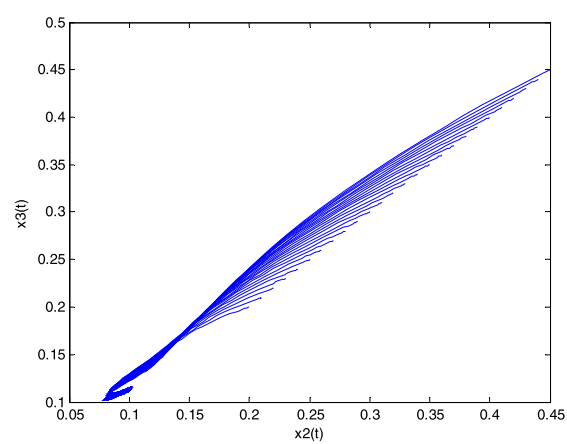

(c)

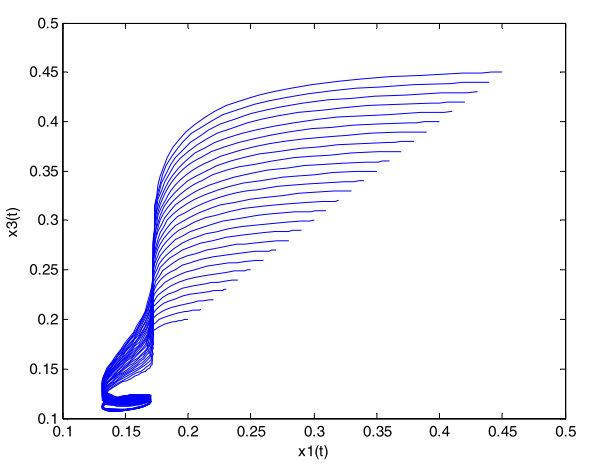

(b)

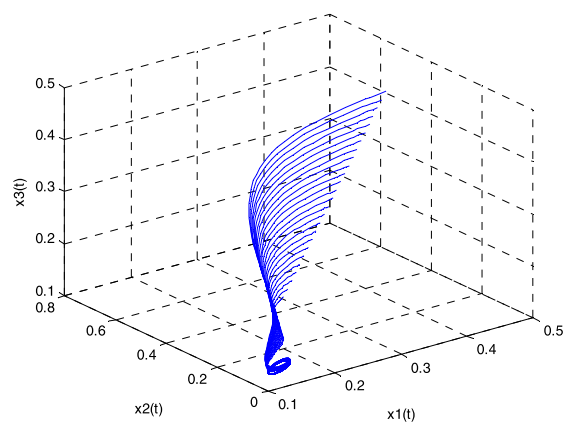

(d)

Figure 3 The dynamic behavior of system (5.1). (a) Phase portrait of $x_{1}(t)$ and $x_{2}(t)$. (b) Phase portrait of $x_{1}(t)$ and $x_{3}(t)$. (c) Phase portrait of $x_{2}(t)$ and $x_{3}(t)$. (d) Phase portrait of $x_{1}(t), x_{2}(t)$, and $x_{3}(t)$

\section{Conclusion}

This paper presents the use of Lyapunov stability theorem and comparison theorem as well as fixed point theory for a system of nonlinear differential equations. This method is a powerful tool for solving nonlinear differential equations in mathematical physics, chemistry, engineering, etc. The technique constructing a suitable Lyapunov function and Poincaré mapping provides a new efficient method to handle the nonlinear structure. 
We have dealt with the problem of positive solution and periodic solution for a LotkaVolterra predator-prey system with ratio-dependent functional responses and feedback controls. The general sufficient conditions have been obtained to ensure the permanence of the system and the global asymptotic stability of a positive solution for the predatorprey system. Furthermore, some conditions for the existence, uniqueness, and stability of a positive periodic solution for the corresponding periodic system are obtained. In addition, some numerical solutions of the equations describing the system are given to illustrate our results. In particular, the sufficient conditions that we obtained are very simple, which provides flexibility for the application and analysis of the Lotka-Volterra predator-prey system.

Remark Obviously, model (1.1) is the extension of model (1.2). Adding delay term to the proposed model (1.1) is our next research work.

\section{Acknowledgements}

The authors would like to thank the referees for their valuable suggestions which helped to improve this work.

\section{Funding}

This work is supported by the Science Fund for Distinguished Young Scholars (cstc2014jc yjjq40004) of China, the Sichuan Science and Technology Program (Grant no. 2018JY0480) of China, the Postdoctoral Science Foundation (Grant no. 2016m602663) of China, the National Nature Science Fund (Project no. 61503053) of China, the Natural Science Foundation Project of CQ CSTC (Grant no. cstc2015jcyj BX0135) of China.

Availability of data and materials

Not applicable.

\section{Competing interests}

The authors declare that they have no competing interests.

\section{Authors' contributions}

CW carried out the study of permanence and global attractivity and participated in the design of the study, YZ carried out the study of periodic solution and participated in the design of the study, YL drafted the manuscript and participated in the design of the study. RL carried out the numerical simulation and helped to draft the manuscript. All authors read and approved the final manuscript.

\section{Author details}

${ }^{1}$ College of Applied Mathematics, Chengdu University of Information Technology, Chengdu, P.R. China. ${ }^{2}$ College of Science, Chongqing University of Posts and Telecommunications, Chongqing, P.R. China. ${ }^{3}$ College of Automation, Chongqing University of Posts and Telecommunications, Chongqing, P.R. China.

\section{Publisher's Note}

Springer Nature remains neutral with regard to jurisdictional claims in published maps and institutional affiliations.

Received: 9 June 2018 Accepted: 13 July 2018 Published online: 21 July 2018

\section{References}

1. Curds, C.R., Cockburn, A.: Studies on the growth and feeding of tetrahymena pyriformis in axenic and monoxenic culture. J. Gen. Microbiol. 54, 343-358 (1968)

2. Hassell, M.P., Varley, G.C.: New inductive population model for insect parasites and its bearing on biological control. Nature 223, 1133-1137 (1969)

3. Salt, G.W.: Predator and prey densities as controls of the rate of capture by the predator didinium nasutum. Ecology $55,434-439$ (1974)

4. Arditi, R., Ginzburg, L.R.: Coupling in predator-prey dynamics: ratio-dependence. J. Theor. Biol. 139, 311-326 (1989)

5. Jost, C., Arino, O., Arditi, R.: About deterministic extinction in ratio-dependent predator-prey models. Bull. Math. Biol. 61, 19-32 (1999)

6. Bianca, C., Pennisi, M., Motta, S., Ragusa, M.A.: Immune system network and cancer vaccine. AlP Conf. Proc. 1389, 945-948 (2011). https://doi.org/10.1063/1.3637764

7. Bianca, C., Pappalardo, F., Motta, S., Ragusa, M.A.: Persistence analysis in a Kolmogorov-type model for cancer-immune system competition. AlP Conf. Proc. 1558, 1797-1800 (2013). https://doi.org/10.1063/1.4825874

8. Kuang, Y.: Rich dynamics of Gause-type ratio-dependent predator-prey system. Fields Inst. Commun. 21, 325-337 (1999)

9. Conser, C., Angelis, D.L., Ault, J.S., Olson, D.B.: Effects of spatial grouping on the functional response of predators, Theor. Popul. Biol. 56, 65-75 (1999) 
10. Kesh, D., Sarkar, A.K., Roy, A.B.: Persistence of two prey-one predator system with ratio-dependent predator influence. Math. Methods Appl. Sci. 23, 347-356 (2000)

11. Pang, P.Y.H., Wang, M.: Strategy and stationary pattern in a three-species predator-prey model. J. Differ. Equ. 200, 245-273 (2004)

12. Baek, S., Ko, W., Ahn, l.: Coexistence of a one-prey two-predators model with ratio-dependent functional responses. Appl. Math. Comput. 219, 1897-1908 (2012)

13. Ko, W., Ahn, l.: A diffusive one-prey and two-competing-predator system with a ratio-dependent functional response: I, long time behavior and stability of equilibria. J. Math. Anal. Appl. 397, 9-28 (2013)

14. Ko, W., Ahn, l.: A diffusive one-prey and two-competing-predator system with a ratio-dependent functional response: II stationary pattern formation. J. Math. Anal. Appl. 397, 29-45 (2013)

15. Sarwardi, S., Haque, M., Mandal, P.K.: Ratio-dependent predator-prey model of interacting population with delay effect. Nonlinear Dyn. 69, 817-836 (2012)

16. Sen, M., BanerJee, M., Morozov, A.: Bifurcation analysis of a ratio-dependent prey-predator model with the Allee effect. Ecol. Complex. 11, 12-27 (2012)

17. Wang, J., Shi, J., Wei, J.: Predator-prey system with strong Allee effect in prey. J. Math. Biol. 62, 291-331 (2011)

18. Zhang, G., Wang, W., Wang, X.: Coexistence states for a diffusive one-prey and two-predators model with B-D functional response. J. Math. Anal. Appl. 387, 931-948 (2012)

19. Zhou, J., Mu, C.: Coexistence of a diffusive predator-prey model with Holling type-II functional response and density dependent mortality. J. Math. Anal. Appl. 385, 913-927 (2012)

20. Mandal, P.S.: Noise-induced extinction for a ratio-dependent predator-prey model with strong Allee effect in prey. Phys. A, Stat. Mech. Appl. 496, 40-52 (2018)

21. Louartassi, Y., Alla, A., Hattaf, K., Nabil, A.: Dynamics of a predator-prey model with harvesting and reserve area for prey in the presence of competition and toxicity. J. Appl. Math. Comput. (2018) https://doi.org/10.1007/s12190-018-1181-0

22. Yin, F.Q., Li, Y.K.: Positive periodic solutions of a single species model with feedback regulation and distributed time delay. Appl. Math. Comput. 153, 475-484 (2004)

23. Chen, F.: Global stability of a single species model with feedback control and distributed time delay. Appl. Math. Comput. 178, 474-479 (2006)

24. Nie, L., Teng, Z., Hu, L., Peng, J.: Permanence and stability in non-autonomous predator-prey Lotka-Volterra systems with feedback controls. Comput. Math. Appl. 58, 436-448 (2009)

25. Chen, F.: The permanence and global attractivity of Lotka-Volterra competition system with feedback controls Nonlinear Anal., Real World Appl. 7, 133-143 (2006)

26. Fan, Y., Wang, L.: Global asymptotical stability of a logistic model with feedback control. Nonlinear Anal., Real World Appl. 11, 2686-2697 (2010)

27. Gopalsamy, K., Weng, P.: Global attractivity in a competition system with feedback controls. Comput. Math. Appl. 45 665-676 (2003)

28. Lai, Y.C., Tel, T.: Transient Chaos: Complex Dynamics on Finite Time Scales. Springer, Berlin (2011)

29. Li, J., Zhao, A., Yan, J.: The permanence and global attractivity of a Kolmogorov system with feedback controls. Nonlinear Anal., Real World Appl. 10, 506-518 (2009)

30. Yang, Z.: Positive periodic solutions of a class of single species neutral models with state dependent delay and feedback control. Eur. J. Appl. Math. 17, 735-757 (2006)

31. Lande, R., Engen, S., Saether, B.E.: Stochastic Population Dynamics in Ecology and Conservation. Oxford University Press, Oxford (2003)

32. Liu, Y., Shan, M., Lian, X.: Stochastic extinction and persistence of a parasite-host epidemiological model. Phys. A, Stat. Mech. Appl. 462, 586-602 (2016)

33. Ridolfi, L., D'Odorico, P., Laio, F.: Noise-Induced Phenomena in the Environmental Sciences. Cambridge University Press, Cambridge (2011)

34. Spagnolo, B., Cirone, M., La Barbera, A., De Pasquale, F.: Noise-induced effects in population dynamics. J. Phys. Condens. Matter 14, 2247-2255 (2002)

35. Spagnolo, B., Fiasconaro, A., Valenti, D.: Noise induced phenomena in Lotka-Volterra systems. Fluct. Noise Lett. 3, L177-L185 (2003)

36. Spagnolo, B., Valenti, D., Fiasconaro, A.: Noise in ecosystems: a short review. Math. Biosci. Eng. 1, 185-211 (2004)

37. Fiasconaro, A., Mazo, J.J., Spagnolo, B.: Noise-induced enhancement of stability in a metastable system with damping Phys. Rev. E, Stat. Nonlinear Soft Matter Phys. 82, 041120 (2010)

38. Ghergu, M., Radulescu, V.: A singular Gierer-Meinhardt system with different source terms. Proc. R. Soc. Edinb., Sect. A $138,1215-1234(2008)$

39. Ghergu, M., Radulescu, V.: Turing patterns in general reaction-diffusion systems of Brusselator type. Commun Contemp. Math. 12,661-679 (2010)

40. Liu, X., Ren, Y., Li, Y.: Four positive periodic solutions of a discrete time Lotka-Volterra competitive system with harvesting terms. Opusc. Math. 31, 257-267 (2011)

41. Giacomoni, J., Hernandez, J., Sauvy, P.: Quasilinear and singular elliptic systems. Adv. Nonlinear Anal. 2, 1-41 (2013)

42. Ghergu, M., Radulescu, V.: Nonlinear PDEs. Mathematical Models in Biology, Chemistry and Population Genetics. Springer Monographs in Mathematics. Springer, Heidelberg (2012)

43. Ciuchi, S., Depasquale, F., Spagnolo, B.: Nonlinear relaxation in the presence of an absorbing barrier. Phys. Rev. E, Stat. Phys. Plasmas Fluids Relat. Interdiscip. Topics 47, 3915-3926 (1993)

44. Bashkirtseva, I., Ryashko, L.: How environmental noise can contract and destroy a persistence zone in population models with Allee effect. Theor. Popul. Biol. 115, 61-68 (2017)

45. Bashkirtseva, I., Ryashko, L.: Noise-induced shifts in the population model with a weak Allee effect. Phys. A, Stat. Mech. Appl. 491, 28-36 (2018)

46. Dubkov, A., Spagnolo, B.: Langevin approach to Lévy flights in fixed potentials: exact results for stationary probability distributions. Acta Phys. Pol. B 38, 1745-1758 (2007)

47. Gao, J.B., Hwang, S.K., Liu, J.M.: When can noise induce chaos? Phys. Rev. Lett. 82, 1132-1135 (1999) 
48. Li, Y., Zhang, T.: Permanence of a discrete $n$-species cooperation system with time-varying delays and feedback controls. Math. Comput. Model. 53, 1320-1330 (2011). https://doi.org/10.1016/j.mcm.2010.12.018

49. Sun, G.Q., Jin, Z., Li, L., Liu, Q.X.: The role of noise in a predator-prey model with Allee effect. J. Biol. Phys. 35, 185-196 (2009)

50. Zhang, X.B., Huo, H.F., Xiang, H., Shi, Q.H., Li, D.G.: The threshold of a stochastic SIQS epidemic model. Phys. A, Stat. Mech. Appl. 482, 362-374 (2017)

51. Shi, Q.H., Wang, S.: Nonrelativistic approximation in the energy space for KGS system. J. Math. Anal. Appl. 462, 1242-1253 (2018)

52. Lu, Z.Q., Liang, G.Z.: Dynamics of a nonautonomous ratio-dependent two competing predator-one prey model. J. Henan Norm. Univ. Nat. Sci. 35(2), 211-214 (2007) (In Chinese)

53. Khalil, H.K.: Nonlinear Systems, 3rd edn. Prentice Hall, Englewood Cliffs (2002)

54. Basener, W.: Topology and Its Applications. Wiley, Hoboken (2006)

Submit your manuscript to a SpringerOpen ${ }^{\circ}$ journal and benefit from:

- Convenient online submission

- Rigorous peer review

- Open access: articles freely available online

- High visibility within the field

- Retaining the copyright to your article

Submit your next manuscript at $\gg$ springeropen.com 